\title{
Ishak Score 5
}

National Cancer Institute

\section{Source}

National Cancer Institute. Ishak Score 5. NCI Thesaurus. Code C95155.

Marked bridg ing fibrosis with formation of occasional hepatic nodules is present. 